$\Rightarrow$ GENETICS

\title{
Weighing up effects of extra chromosomes
}

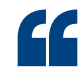

likely contextdependency of chromosomal alterations
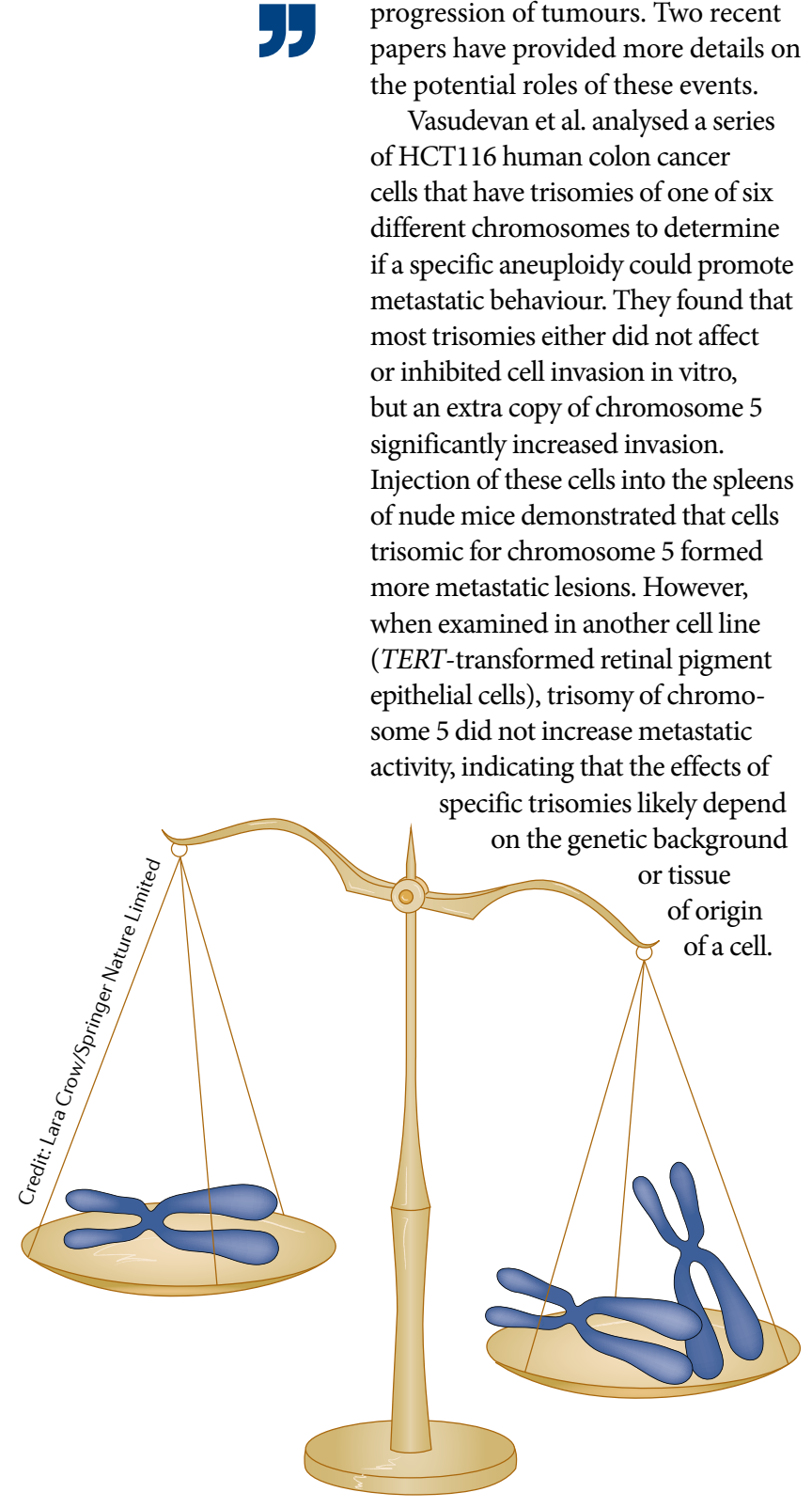

Aneuploidy of one or more chromosomes and whole-genome doubling (WGD) are both often observed in cancer genomes. However, little is known about the effects these chromosomal changes have on the evolution and progression of tumours. Two recent papers have provided more details on the potential roles of these events.

Vasudevan et al. analysed a series of HCT116 human colon cancer cells that have trisomies of one of six different chromosomes to determine if a specific aneuploidy could promote metastatic behaviour. They found that most trisomies either did not affect or inhibited cell invasion in vitro, but an extra copy of chromosome 5 significantly increased invasion. Injection of these cells into the spleens of nude mice demonstrated that cells trisomic for chromosome 5 formed more metastatic lesions. However, when examined in another cell line (TERT-transformed retinal pigment epithelial cells), trisomy of chromosome 5 did not increase metastatic activity, indicating that the effects of specific trisomies likely depend
The gain of chromosome 5

par
analyse this in more detail, they used pan-cancer data from The Cancer Genome Atlas (TCGA) to compare aneuploidy with outcomes. Patients with highly aneuploid tumours had worse outcomes than those with low aneuploidy. Looking at specific chromosomes, they found that sometimes a gain of one chromosome could be informative for prognosis, even if overall aneuploidy was not. In addition, most gains or losses of a chromosome correlated with lower patient survival, but some correlated with improved survival. Finally, the data indicated that aneuploidies that suppress invasive behaviour are likely selected against during tumour development.

López et al. investigated the effects of WGD during the evolution of non-small cell lung cancer (NSCLC) using data from the TRACERx study as well as from TCGA. WGD is a frequent event in tumours and is associated with poor prognosis. The authors hypothesized that there might be selective pressures favouring genome doubling during tumorigenesis.

Simulations aimed at understanding how WGD might affect cancer cell viability suggested that WGD might help buffer deleterious effects, such as homozygous disruption of essential genes. In support of this model, analysis of sequencing data uncovered evidence of negative selection before but not after WGD in tumours. The authors also found similar patterns in other cancer types and showed that tumours with high loss of heterozygosity (LOH) more frequently have high rates of WGD.

Looking closer at regions of LOH that occurred before WGD, the authors noted that these regions contained many known tumour suppressors, such as TP53 and PTEN, which exhibited both LOH and mutation. They then used this information to try and identify previously unknown tumour suppressors and found ZNF750, NOTCH1 and SMAD4 as likely candidates in squamous cell lung cancer (a type of NSCLC).

These two papers indicate that changes in chromosome numbers have wide-ranging effects, and that much remains to be learnt about their roles in cancer progression.

Sarah Seton-Rogers

ORIGINAL ARTICLES López, S. et al. Interplay between whole-genome doubling and the accumulation of deleterious alterations in cancer evolution. Nat. Genet. 52, 283-293 (2020)| Vasudevan, A. et al. Single-chromosomal gains can function as metastasis suppressors and promoters in colon cancer. Dev. Cell 52, 413-428 (2020) 